\title{
Entropy and phase transitions in partially ordered sets
}

\section{Deepak Dhar}

Downs Laboratory of Physics, California Institute of Technology, Pasadena, California 91125

(Received 16 January 1978)

\begin{abstract}
We define the entropy function $S(\rho)=\lim _{n \rightarrow \infty} 2 n^{-2} \ln N(n, \rho)$, where $N(n, \rho)$ is the number of different partial order relations definable over a set of $n$ distinct objects, such that of the possible $n(n-1) / 2$ pairs of objects, a fraction $\rho$ are comparable. Using rigorous upper and lower bounds for $S(\rho)$, we show that there exist real numbers $\rho_{1}$ and $\rho_{2} ; .083<\rho_{1} \leq 1 / 4$ and $3 / 8 \leq \rho_{2}<48 / 49$; such that $S(\rho)$ has a constant value $(\ln 2) / 2$ in the interval $\rho_{1} \leq \rho \leq \rho_{2}$; but is strictly less than $(\ln 2) / 2$ if $\rho \leq .083$ or if $\rho \geq 48 / 49$. We point out that the function $S(\rho)$ may be considered to be the entropy function of an interacting "lattice gas" with long-range three-body interaction, in which case, the lattice gas undergoes a first order phase transition as a function of the "chemical activity" of the gas molecules, the value of the chemical activity at the phase transition being 1. A variational calculation suggests that the system undergoes an infinite number of first order phase transitions at larger values of the chemical activity. We conjecture that our best lower bound to $S(\rho)$ gives the exact value of $S(\rho)$ for all $\rho$.
\end{abstract}

In this paper we discuss the asymptotic enumeration of partial order relations defined over a set of $n$ distinct objects when a finite fraction $\rho$ of the $n(n-1) / 2$ pairs are comparable.

Let $N(n)$ be the total number of partial order relations defined over $n$ objects. It is easy to show that

$$
N(n) \geqslant 2^{n^{2} / 4} \text {. }
$$

Kleitman and Rothschild ${ }^{1}$ have shown that

$$
\ln N(n) \leqslant \frac{n^{2}}{4} \ln 2+A n^{3 / 2} \ln n,
$$

for some finite constant $A$. Combining these two results we see that

$$
\lim _{n \rightarrow \infty} \frac{2}{n^{2}} \ln N(n)=\frac{1}{2} \ln 2 .
$$

We are here interested in a more detailed asymptotic enumeration of partial order relations. For this purpose, we define the function

$$
S(\rho) \stackrel{\text { deff }}{=} \lim _{n \rightarrow \infty} 2 n^{-2} \ln N(n, \rho),
$$

where $N(n, \rho)$ is the number of partial order relations on $n$ distinct objects such that $\rho n(n-1) / 2$ of the $n(n-1) / 2$ pairs are comparable. [We call a pair $(a, b)$ comparable if $a>b$ or $b>a$. ] If $\rho n(n-1) / 2$ is not integral, we round it off to the nearest integer. The difference is clearly unimportant for large $n$. Clearly we have

$$
S(0)=S(1)=0,
$$

and from (2)

$$
S(\rho) \leqslant \frac{1}{2} \ln 2 .
$$

Our first results about $S(\rho)$ are given in Theorem 1 .

Theorem 1: (i) $S(\rho) / \rho$ is a monotonic nonincreasing function of $\rho$. of $\rho$.

(ii) $S(\rho) /(1-\rho)$ is a monotonic nondecreasing function

Proof: (i) From any partial order relation on $n$ objects, we can generate a partial order relation on $(n+\epsilon)$ objects by introducing $\epsilon$ new elements, incomparable to all of the $n$ elements and to each other. We thus have the trivial inequality

$$
N(n, \rho) \leqslant N\left(n+\epsilon, \frac{\rho n(n-1)}{(n+\epsilon)(n+\epsilon-1)}\right) .
$$

The theorem follows if we take the logarithms of both sides and the limit of $n$ and $\epsilon$ going to inf inity.

(ii) The proof is similar to that of (i). Add of a chain of $\epsilon$ new elements to the original set of $n$ elements such that any of the new $\epsilon$ elements is less than any of the original $n$ elements. The density of comparable pairs in this new set of $(n+\epsilon)$ elements is

$$
\rho^{\prime}=\frac{2}{(n+\epsilon)(n+\epsilon-1)}\left(\frac{n(n-1)}{2} \rho+n \epsilon+\frac{\epsilon(\epsilon-1)}{2}\right) .
$$

The result follows from the inequality

$$
N\left(n+\epsilon, \rho^{\prime}\right) \geqslant N(n, \rho),
$$

by taking the logarithms of both sides and going to the limit of large $n$ and $\epsilon$.

We note that Theorem 1 implies that $S(\rho)$ is a continuous function of $\rho$. It is quite likely that the results of this theorem can be made stronger. In particular, we would like to prove that $S(\rho)$ is a convex function of $\rho$. At the present time, however, the convexity of $S(\rho)$ is an unproved conjecture. We now derive a lower bound for $S(\rho)$.

Theorem 2: Let $f_{k}, p$ be any positive real numbers satisfying the following conditions:

(i) $f_{i} \geqslant 0$,

(ii) $0 \leqslant p \leqslant 1$,

(iii) $\sum_{i} f_{i}=1$,

(iv) $\sum_{i}^{i}\left[f_{i}^{2}+2 f_{i} f_{i+1}(1-p)\right]=1-\rho$.

Then

$$
S(\rho) \geqslant 2\left(\sum_{i} f_{i} f_{i+1}\right)[-p \ln p-(1-p) \ln (1-p)],
$$

Proof: We consider a set of $n$ distinct elements where $n$ is very large. This may be divided into disjoint subsets so that the $i$ th subset contains $n f_{i}$ elements. (For simplicity, we assume that $n f_{i}$ are all integers. This is clearly inessential as we let $n$ tend to infinity in the end.) We now construct a partial order relation amongst these objects as follows: 
1. Any element in the $i$ th subset is greater than any element in the $j$ th subset if $j>i+1$.

2. Elements belonging to the same subset are noncomparable.

3 . In no case is an element in the $(i+1)$ th subset greater than an element in the $i$ th subset.

Any relation which satisfies these conditions is a partial order relation. To complete the construction, we have to specify the relation between the $n^{2}\left(\sum_{i} f_{i} f_{i+1}\right)$ $=N_{1}$ (say) pairs of the type $(a, b)$, where $a$ and $b$ belong to the $i$ th and $(i+1)$ th subsets repectively for some $i$. We arbitrarily set $a>b$ for $p N_{1}$ of these pairs and $a$ incomparable to $b$ for the rest. The resulting relation has a fraction $\rho$ of all the pairs comparable.

Total no。 of such relations $={ }^{N_{1}} C_{p N_{1}} \leqslant N(n, \rho)$. Taking the logarithms and going to the limit of large $n$, we get Theorem 2 .

Corollary 2. 1: If $\frac{1}{4} \leqslant \rho \leqslant \frac{3}{8}$, then $S(\rho)=\frac{1}{2} \ln 2$.

Proof: Choose

$$
f_{1}=\frac{1}{4}+\left(\frac{3}{16}-\frac{\rho}{2}\right), \quad f_{2}=\frac{1}{2}, f_{3}=\frac{1}{4}-\left(\frac{3}{16}-\frac{\rho}{2}\right)^{1 / 2}, p=\frac{1}{2} \text {. }
$$

Then Theorem 2 gives us $S(\rho) \geqslant \frac{1}{2} \ln 2$ 。

Combined with Eq. (6), this proves the corollary.

Corollary 2.2: If $\rho \leqslant \frac{1}{4}$; then $S(\rho) \geqslant \frac{1}{2}[-2 \rho \ln (2 \rho)-(1$ $-2 \rho) \ln (1-2 \rho)$ ]

Proof: Put $f_{1}=f_{2}=\frac{1}{2}, p=2 p$ in Theorem 2.

Corollary 2.3: If $\rho>\frac{3}{8}$, then $S(\rho) \geqslant \frac{4}{5}(1-\rho) \ln 2$.

Proof: This follows from Theorem 1 by putting $S\left(\frac{3}{8}\right)$ $=\frac{1}{2} \ln 2$

We can determine better lower bounds for $S(\rho)$ than given by Corollary 2.3 by using Theorem 2 and variational calculus to choose $f_{i}$ so that the largest lower bound is attained. Using Lagrange's multipliers, it is easy to show that the optional choice of $\left\{f_{i}, p\right\}=\left\{f_{i}^{*}, p^{*}\right\}$ satisf ies the conditions

$$
f_{i}^{*}\left(\frac{\ln \left(1-p^{*}\right)}{\ln p^{*}}-1\right)-f_{i-1}^{*}-f_{i+1}^{*}\left\{\begin{array}{l}
=M, \text { if } f_{i}^{*}>0, \\
\geqslant M, \text { if } f_{i}^{*}=0 .
\end{array}\right.
$$

$p^{*}$ and $M$ are chosen so that the corresponding solution $\left\{f_{i}^{*}\right\}$ satisf ies conditions (i)-(iv) of Theorem 2. This determines $p^{*}$ uniquely for a given $\rho$. If however

$$
\frac{\ln \left(1-p^{*}\right)}{\ln p^{*}}-1=2 \cos \frac{2 \pi}{r}, r=4,5,6 \cdots ;
$$

then the corresponding solution $\left\{f_{i}^{*}\right\}$, and hence $\rho$, is not unique for a given value of $p^{*}$. The graph of $p^{*}$, as a function of $\rho$, shows intervals of $\rho$ for which the value of $p^{*}$ is a constant. It is easy to verify that in each of these intervals $S^{*}(\rho)$, our best lower bound to $S(\rho)$, is a linear function of $\rho$.

It is quite plausible that the optimal lower bound given by Theorem 2 gives us the exact value of $S(\rho)$. The only partial order relations not counted in Theorem 2 are those containing at least one incomparable $(a, b)$ such that $a$ and $b$ belong to the $i$ th and $j$ th subsets respectively with $j>i+1$, for some $i, j$. This however implies that no element of the $(i+1)$ th subset is simultaneously comparable to both $a$ and $b$; and the probability of such an event tends to zero exponentially for large $n$. We conjecture that the best lower bound to $S(\rho)$ given by Theorem 2, coincides with the exact value of $S(\rho)$ for all $\rho$.

We now obtain upper bounds for $S(0)$, which are stronger than (6) in some interval of $\rho$.

If $\rho$ is very small, it is easy to see that

$$
S(\rho) \leqslant \rho \ln 2-\rho \ln \rho-(1-\rho) \ln (1-\rho) \text { 。 }
$$

To prove this, we just observe that there are ${ }_{n(n-1) / 2} C_{n(n-1) \rho / 2}$ ways of choosing $\rho n(n-1) / 2$ comparable pairs out of $n(n-1) / 2$, and there are at most two possibilities of ordering for each comparable pair. Taking the logarithms and limit of large $n$ gives us (12). In particular, we note that (12) implies that

$$
S(6)<\frac{l}{2} \ln 2 \text { if } \rho<.083 \text { 。 }
$$

While (12) gives a fairly good upper bound if $\rho$ is very small, it is quite worthless if $\rho$ is close to 1 and $(1-\rho)$ is small. In this case a better upper bound is given by the following theorem:

Theorem 3: $S(\rho) \leqslant 4 \ln 2(1-\rho)^{1 / 2}$.

Proof: We note that the maximum number of mutually noncomparable objects in a partial order relation on $n$ objects with $\rho n(n-1) / 2$ comparable pairs is less than $n(1-\rho)^{1 / 2}+1=m$ (say). Hence, by Dilworth's theorem, ${ }^{2}$ we can choose $m$ chains such that their union contains all the $n$ elements.

Let the lengths of these chains be $l_{1}, l_{2}, l_{3} \ldots, l_{m}$ in decreasing order of magnitude. Consider now any two chains $i$ th and $j$ th. Let $N_{i j}$ be the number of different ways we may assign a partial order relation on the set formed by the union of these chains consistent with their chain structure. Then we have

$$
\left.N_{i j} \leqslant\left[\left(l_{i}+l_{j}\right) ! / l_{i} ! l_{j} !\right)\right]^{2} \text {. }
$$

This may be seen as follows: Let the elements of the $i$ th chain be $a_{1}>a_{2}>a_{3} \cdots>a_{l_{j}}$, and the elements of the $j$ th chain be $b_{1}>b_{2} \cdots b_{l_{f}}$. Then a partial order relation over the combined set of $\left(l_{i}+l_{j}\right)$ elements is uniquely specified by a list of $\left(l_{i}+2 l_{j}\right)$ elements. $a_{\alpha}, b_{\beta}^{\prime}$ and $b_{\beta}^{\prime \prime}$ $\left(\alpha=1\right.$ to $l_{i}, \beta=1$ to $\left.l_{j}\right)$ of the type

$$
a_{1} a_{2} b_{1}^{\prime} a_{3} b_{2}^{\prime} a_{4} a_{5} b_{3}^{\prime} b_{1}^{\prime \prime} \cdots \text {. }
$$

In this list $b_{\beta}^{\prime}$ occurs after all the elements of the $i$ th chain which are greater than $b_{\beta}$, and before all the elements of the $i$ th chain which are not. Similarly $b_{\beta}^{\prime \prime}$ occurs after all elements of the $i$ th chain which are not less than $b_{\beta}$, and before all elements that are. Clearly if $\beta_{1}-\beta_{2}$, then $b_{\beta_{1}}^{\prime}$ occurs after $b_{\beta_{2}}^{\prime}$ in the list, and $b_{\beta_{2}}^{\prime \prime}$ occurs after $b_{\beta_{2}}^{\prime \prime}$. We further assume that if in this list, there is an uninter rupted string of $b$ 's, then all $b_{\beta}^{\prime \prime}$ 's occur after $b_{\beta}^{\prime}$ 's in that string。

The number of ways we may insert a chain of $b_{\beta}^{\prime}$ 's in the chain of $a^{\prime} \mathrm{s}$ is $\left(l_{i}+l_{j}\right) ! /\left(l_{i} ! l_{j} !\right)$. Similarly for $b_{\beta}^{\prime \prime}$ 's. Hence the total number of such lists is equal to 


$$
\left[\left(l_{i}+l_{j}\right) ! / l_{t} \mid l_{j} !\right]^{2}
$$

Not all these lists correspond to partial order relations. In particular, $b_{\beta}^{\prime}$ must precede $b_{\beta}^{\prime \prime}$ in the list for all $\beta$, for the list to correspond to a partial order relation. This proves (14).

Now, the number of ways $m$ disjoint chains may be chosen out of $n$ elements is ${ }^{n+m-1} P_{n}$. Hence the total number of partial order relations having at most $m$ noncomparable elements is

$$
\leqslant{ }^{n+m-1} P_{n} \max _{\left\langle l_{i}\right\}}\left[\prod_{\substack{i, j=1 \\ i<j}}^{m} \frac{\left(l_{i}+l_{j}\right) !}{l_{i} ! l_{j} !}\right]^{2}
$$

where the maximum value of the term inside the square brackets is to be taken over all $m$ partitions of $n$ (i. e., $\left.\sum_{i=1}^{m} l_{i}=n\right)$. The maximum is attained if all $l_{i}$ are equal to $n / m$. We drop here the constraint of $l_{i}$ being integers. Taking the logarithm of the resulting inequality and retaining only the terms of order $n^{2}$, we get

$$
\frac{n^{2}}{2} S(\rho) \leqslant \frac{n^{2}}{2}(1-\rho)^{1 / 2} 4 \ln 2+O\left(n^{2}\right) .
$$

Taking the limit $n \rightarrow \infty$, we obtain

$$
S(\rho) \leqslant(1-\rho)^{1 / 2} 4 \ln 2 .=
$$

Corollary 3. 1: If $\rho>48 / 49$, then, $S(\rho)<(\ln 2) / 2$.

Proof: From Theorem 3, $S(48 / 49) \leqslant(2 / 49) \ln [14 ! /(7$ ()$\left.^{2}\right]<\frac{1}{2} \ln 2$

Theorem 3 is not the best possible. Of all the possible decomposition of a partial order relation into chains; we may choose the one which gives the largest value of $\sum_{i=1}^{m} l_{i}{ }^{2}$. (Some of the $l_{i}$ 's may be zero.) Then each of the elements of the $i$ th chain is incomparable to at least one element in each of the preceding chains $j<i$. This give the inequality

$$
\sum_{i=1}^{m}(i-1) l_{i} \leqslant(1-\rho) n(n-1) / 2 .
$$

This constraint, in addition to sharper bounds on $N_{i j}$, may be used to obtain an improved upper bound to $S(\rho)$. These bounds are, however, still far above the true value of $S(\rho)$. In any case, Theorem 3 is quite sufficient to prove that $S(\rho)$ is nonanalytic.

Putting together the results of this paper, we see that $S(\rho)$ is a continuous function of $\rho$ in the allowed range of variation of $\rho, 0 \leqslant \rho \leqslant 1$. It, however, is a nonanalytic function of $\rho$, and there exist numbers $\rho_{1}$ and $\rho_{2}$ such that

$$
S(\rho)=\frac{1}{2} \ln 2 \text { for } \rho_{1} \leqslant \rho \leqslant \rho_{2} .
$$

We have shown that $S(\rho)$ is strictly less than $\frac{1}{2} \ln 2$, if $\rho \leqslant .083$ or if $\rho \geqslant 48 / 49$. Hence we get from Corollary 2.1

$$
.083 \leqslant \rho_{1}<\frac{1}{4},
$$

and

$$
\frac{3}{8} \leqslant \rho_{2}<48 / 49 \text {. }
$$

We may interpret $S(\rho)$ to be the entropy per particle of an interacting "lattice gas." Here the "lattice sites" are the $n(n-1) / 2$ pairs of elements. The three possible states $(a>b, a<b$ or a $\not \not b)$ of a pair $(a, b)$ under a reflexive antisymmetric binary relation correspond to three possible "states" of a lattice site in the interacting gas language. A "configuration" of the 'gas' corresponds to a reflexive, antisymmetric binary relation on $n$ elements, and is specified by specifying the "state" of eqch "lattice site." We call the $(a \not R b)$ state the "unoccupied" state of the lattice site $(a, b)$. $a>b$ and $a<b$ correspond to two different possible states of the gas molecule at the "occupied site" $(a, b)$.

The transititivity property of the partial order relations corresponds to a 3 -body interaction between lattice sites. The interaction is hard-core type, in the sense that it excludes certain configurations from the statistical sum, or alternatively puts their weight equal to zero. This condition may be relaxed and the properties of soft core systems may be of interest.

The flat portion of the $S(\rho)$ curve corresponds to a first order phase transition in the interacting lattice gas. The corresponding value of the chemical potential of the lattice gas is zero $\mu=-\partial S / \partial \rho$. This corresponds to the chemical activity of the gas being 1 . We may speak of the states with $\rho<\rho_{1}$ and $\rho>\rho_{2}$ constitut ing the 'disordered phase' and the 'ordered phase' respectively, In the language of partial order relations, the ordered phase is characterized by a larger value of "average maximal chain length。"

The greatest lower bound approximation to $S(\rho)$, as given by Theorem 2 , shows that $S(\rho)$, as a function of $\rho$, contains an infinite number of linear segments. In the language of phase transitions, the system exhibits an infinite number of first order phase transitions. The different phases corresponds to different values of "average maximal chain length," which serves the role of the order parameter in this system. The order parameter jumps by one unit across a phase transition.

The relationship of these phase transitions to phase transitions in realistic physical systems, if any, is not very clear. The transitions are governed by the strong, long range nature of the 3 -body interaction here, not usually encountered in physical systems. While the asymptotic enumeration of partial order relations is of sufficient interest intrinsically, the study of the mechanism of these transitions may be of some interest in statistical physics. In particular, the distribution of zeros of the grand partition function ${ }^{3,4}$ of this system may be of some interest.

I would like to thank Professor Jon Mathews for many critical comments, Professor Kenneth Bogart for pointing out Ref. 1 to me, and Ms. RLou Norquist for typing the manuscript.

${ }^{1}$ D. Kleitman and B. Rothschild, Proc. Amer. Math. Soc. 25, $276(1970)$.

${ }^{2}$ R. P. Dilworth, Ann. Math. 51, 161 (1950).

${ }^{3}$ C. N. Yang and T. D. Lee, Phys, Rev, 87, 404 (1952).

${ }^{4}$ K. Ikeda, Prog. Theor. Phys. 52, 54 (1974). 